\title{
La guerra per il Mezzogiorno. Italiani, borbonici e briganti 1860-1870
}

\author{
di Chiara Nencioni
}

Mar 17, 2021 | In evidenza, Uno sguardo su $|\underline{0}|$

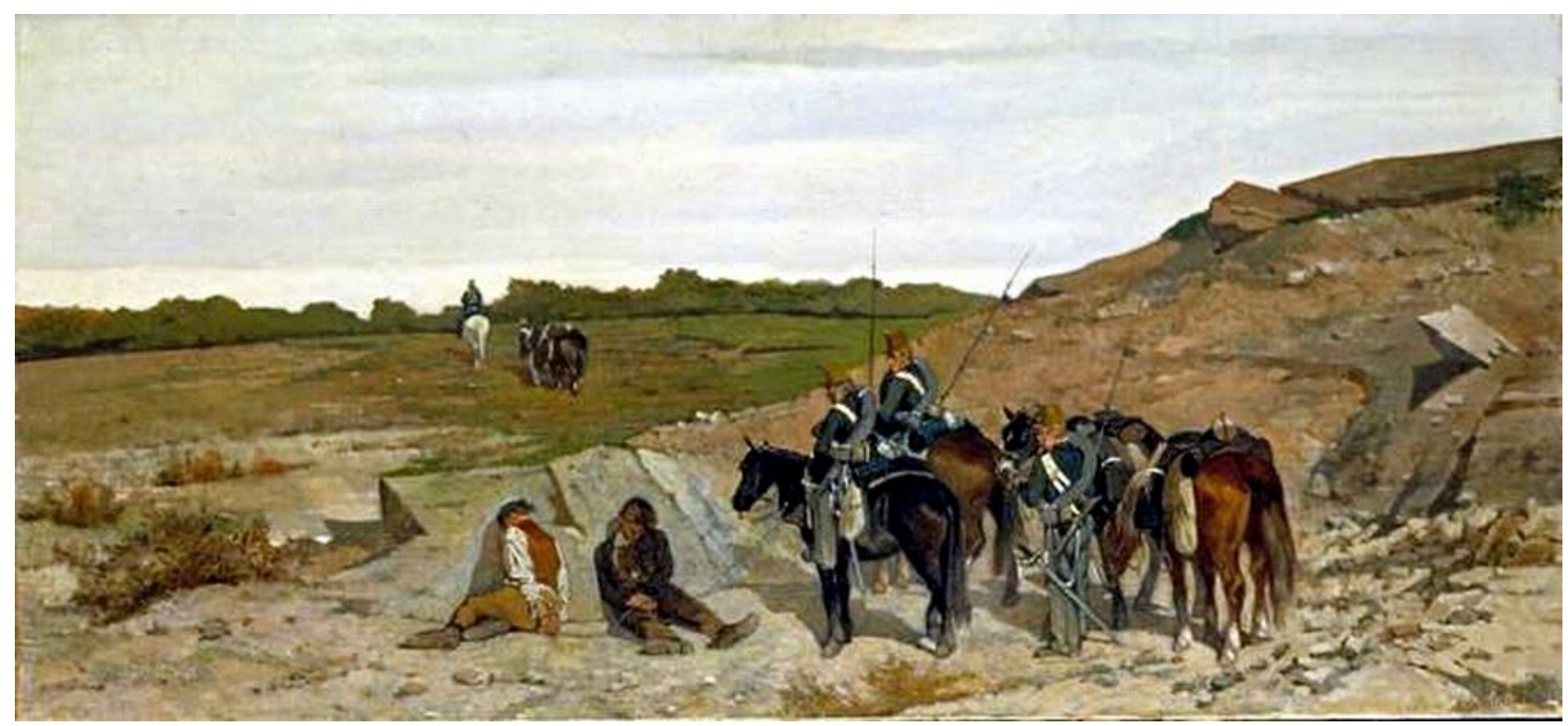

Di Giovanni Fattori - http://it.wahooart.com/@ @/8LJ2UT-Giovanni-Fattori-Episodio-nellacampagna-contro-il-brigantaggio, Pubblico dominio, Collegamento

\section{Carmine Pinto}

\section{La guerra per il Mezzogiorno. Italiani, borbonici e briganti 1860-1870 Laterza, Roma-Bari 2019}

Il brigantaggio fu l'eroica resistenza meridionale al colonialismo sabaudo o la sfida allo Stato di bande criminali? Questa è la domanda che leggiamo nella quarta di copertina del libro di Carmine Pinto, professore ordinario di Storia contemporanea presso 1'Università degli studi di Salerno. Il saggio, dal titolo La guerra per il Mezzogiorno. Italiani, borbonici e briganti 1860-1870, edito da Laterza nel maggio 2019 per la collana Cultura storica, fornisce una risposta motivata a tale domanda attraverso una vastissima documentazione storica e archivistica. Ogni episodio trattato è ampiamente documentato, tracciabile grazie a delle note dettagliate che si trovano in settanta pagine in calce al libro. Inoltre, per potere collocare gli eventi in un quadro geografico di preciso riferimento, il volume è corredato di otto cartine topografiche. Proprio l'estrema acribia è valsa all'autore diversi premi dedicati alla saggistica (Premio Sissco 2020, il libro è stato fra i tre finalisti del Premio Friuli Storia nello stesso anno).

Il saggio si inserisce pienamente nel dibattito politico-culturale di crescente attualità sul neoborbonismo, sfrondandone gli allori epici e la mistificazione della figura del brigante.

La guerra per il Mezzogiorno, in gran parte delle ricostruzioni post unitarie, è stata considerata un effetto collaterale dell'inevitabile processo unitario; nel secondo dopoguerra ha prevalso invece una interpretazione in chiave di conflitto sociale o di repressione criminale; più recentemente è fiorito il mito del regno borbonico come realtà felice e progredita, stroncata e depredata da 
un 'invasione sabauda. Un mito alimentato da una pubblicistica molto popolare che ha trovato il suo brodo di coltura nel perdurante divario tra nord e sud del nostro paese generando una sorta di patriottismo rivendicativo.[1]

Quella del meridione è «una guerra antica»[2], che affonda le sue radici alla fine del Settecento, con l'arrivo della flotta da guerra francese nel Golfo di Napoli nel dicembre del 1792. Con la Restaurazione e il Congresso di Vienna religione e politica si sovrappongono nel borbonismo e Ferdinando II si presenta come il campione della reazione assolutista, sostenitore di un equilibrio su base dinastico-conservatrice che, anche per questo, ha goduto sempre dell' appoggio della Chiesa. Il Regno Sabaudo si colloca invece nel sistema continentale di Napoleone III rafforzando anche il rapporto con l'impero inglese. Cavour facilita l'amalgama tra patriottismo monarchico-sabaudo e movimento nazionale.

Il mito di Garibaldi è al suo apice nel meridione d'Italia, il Regno delle Due Sicilie è finito e la rivoluzione italiana è festeggiata a Napoli. L'esercito sabaudo, con il re in testa, invade le Marche; il mondo intellettuale, militare e istituzionale unitario si attiva massicciamente per il plebiscito. Ma nel meridione la tensione cresce. Inizia così una guerra tra unificazione e controrivoluzione che durerà quasi un decennio. Il conflitto cambia di segno, e alla campagna tra eserciti regolari e volontari subentra la guerriglia rurale, accompagnata da una massiccia guerra di propaganda, che coinvolge largamente la popolazione civile. I borbonici oppongono le tradizioni di indipendenza del Regno garantite dalla monarchia napoletana e della Chiesa. La controrivoluzione borbonica fallisce, ma seleziona quadri del futuro brigantaggio politico.

È un passaggio che Pinto ricostruisce quasi con un andamento annalistico, registrando giorno per giorno, ad eccezione che nei capitoli sesto e settimo, gli scontri fra bande ed esercito sabaudo. Il reclutamento dei briganti borbonici fa breccia tra gli ex militari, cui si aggregano contadini, fuorilegge, gente attratta dalla avventura, dal bottino, dalla fedeltà al re, tutti uomini di bassa estrazione sociale insoddisfatti della propria condizione e giovani animosi, per i quali l'insurrezione diventa un'occasione di fare bottino e di ottenere promozione sociale. Comitati clandestini forniscono ai briganti denaro, armi e reclute e sviluppano una campagna di propaganda di ampio respiro.

Contro i briganti parte un reggimento proveniente dal territorio italiano già unificato, cui si unisce poi, dato l'impegno pluriennale, una guardia nazionale con ufficiali di tutta la penisola. Le prime azioni dei briganti coincidono con forme abituali di violenza rurale: rapine e assalti nelle vie di passaggio. Il conflitto subito infrange i limiti della guerra regolare, registrando livelli di brutalità e di violenza che non erano stati toccati mai nelle campagne risorgimentali: omicidi a sangue freddo, abitudine alla violenza, unitari che fucilano in pubblico i banditi rifiutando loro qualsiasi tipo di riconoscimento come belligeranti. I briganti non sono di certo dei Robin Hood che rubano ai ricchi per dare ai poveri, e i loro capi non sono dei Che Guevara: Pinto sfata il mito in voga del brigantaggio meridionale simbolo di libertà e di ribellione nei confronti degli invasori piemontesi, e respinge le interpretazioni forzate che lo considerano una forma di lotta sociale dei contadini contro i padroni. In realtà i gruppi armati vivono e si finanziano a spese del territorio, il controllo della popolazione è l'elemento strategico fondamentale, e la guerra delle bande va man mano assumendo un volto sempre più violento: $\mathrm{i}$ capi sanno che la crudeltà gioca a loro vantaggio per spaventare $\mathrm{i}$ civili e scatenare una reazione alla cieca del nemico.

Il Mezzogiorno - a esclusione della Sicilia, le cui élite si sono schierate nella stragrande maggioranza col nuovo stato - è diviso: da un lato è cruciale la presenza di Vittorio Emanuele II, perché nel sud monarchico solo la sostituzione di un re con un altro garantisce la massiccia adesione del notabilato locale che chiede sicurezza; dall'altro la reazione cattolica amplifica 
l'opposizione allo Stato italiano. Le istituzioni ecclesiastiche dichiarano incompatibile con la coscienza cattolica il giuramento per Vittorio Emanuele II, e Pio IX dà pieno appoggio e asilo a Francesco II e ne garantisce, insieme a Napoleone III, la sicurezza personale. Pinto inserisce la guerra per il meridione nel quadro della politica europea: alcuni paesi nella prima fase restano al fianco di Francesco II, la Baviera, l'Austria e soprattutto la Russia (sebbene per soli due anni). Altre potenze, invece, riconoscono prontamente il Regno d'Italia: la Gran Bretagna, la Francia poi anche il Brasile, l'Impero ottomano, il Belgio, la Prussia e, nel 1864, persino il re di Spagna abbandona il cugino borbonico. L'autore in conclusione definisce il brigantaggio «una degenerazione sociale, strumentalizzata da borbonici e clericali».

Pinto dedica l'intero capitolo quinto al 1862, un anno di crisi: la politica di integrazione tentata dagli Italiani viene sommersa dalla offensiva del brigantaggio politico. Destra e sinistra si scontrano sul tema della piemontesizzazione e una delegazione guidata da Crispi chiede un intervento aggressivo contro il clero e su Roma, proponendo addirittura il ritorno di Garibaldi contro i briganti. La grande offensiva del 1862 non ottiene risultati militari strategici, ma contribuisce enormemente alla grave crisi politica e civile del movimento nazionale italiano. Le forze italiane si misurano con azioni sperimentate da secoli nelle campagne meridionali: imboscate, fughe, assalti a sorpresa. Gli ufficiali scoprono i briganti perfettamente «versati nella conoscenza pratica del terreno». Nel 1863 la legge Pica concede provvedimenti straordinari puntando alla completa legittimazione della repressione al brigantaggio, e questo comporta enormi perdite fra i briganti.

Pinto sottolinea come nel fenomeno complessivo fossero riconoscibili due guerre parallele: «la guerra di idee» e «la guerra di combattenti». La prima si basa sul potere comunicativo degli unitari, che si avvalgono di libri, giornali e periodici, dipinti, fotografie, canzoni, satire, monumenti; sfrutta il ruolo crescente delle arti visive come strumenti di largo impatto, capaci di raggiungere una ampia diffusione. La propaganda borbonica, invece, fonde religione e popolo; presenta i briganti come una resistenza popolare capace di sfidare l'esercito invasore e i suoi tirapiedi, interpretando gli autentici valori della nazione napoletana e del re, bolla la repressione delle rivolte come «una guerra di sterminio» e di spoliazione. L'autore afferma inoltre che «la guerra dei briganti ha registrato la quasi completa assenza di distinzione tra conflitto politico, attività criminale e scopi privati», e smonta la visione romantica del brigante propria delle narrazioni popolari.

Nel 1864 ci sono ormai due guerre completamente differenti: da un lato gli Italiani combattono per distruggere definitivamente il brigantaggio e stabilizzare lo Stato, dall'altro i briganti cercano solo di sopravvivere mettendosi in salvo nella rete di appoggi borbonica. Nell'inverno di quello stesso anno si determina il collasso finale, anche se nell'estate del 1865 i superstiti del brigantaggio tentano la loro ultima offensiva. Nel 1867 il conflitto con i briganti e i Borboni è vinto ma non concluso: nel 1870 sono catturati e uccisi gli ultimi capi importanti, due anni dopo pochissime bande sono ancora in circolazione. Nel 1874 il governo Minghetti chiude le residue zone di guerra attive contro i briganti, e a partire dal 1880 il brigantaggio si trasforma in un mito, i briganti sono ormai oggetto di ricerche e di leggende.

Per la ricchezza di dati, fonti, indicazioni topografiche, il saggio di Pinto non è facilmente fruibile direttamente dagli studenti, ma è uno strumento prezioso nelle mani dei docenti per affrontare varie tematiche relative e alla disciplina di educazione civica.

Una modalità di lavoro consiste, ad esempio, nel prendere spunto dall'attualità: il Consiglio regionale della Puglia ha approvato, quasi all'unanimità, il 4 luglio 2017 una mozione del Movimento 5 Stelle che impegna il presidente e la giunta di centrosinistra «a indicare il 13 febbraio (il 13 febbraio 1861 si arrese Gaeta, ndr) come giornata ufficiale in cui si possano commemorare i meridionali che perirono in occasione dell'unità, nonché i relativi paesi rasi al suolo». Da questa delibera regionale può scaturire un dibattito sul proliferare di giorni di memoria e di ricordo per 
svariati motivi e sulla efficacia della istituzionalizzazione di certe ricorrenze per preservarne realmente la memoria.

Utile, inoltre, lavorare sul «paradigma vittimario», palesando l'istituzionalizzazione di mitografie "sudiste"[3] che riattualizza il canone nazionalista ottocentesco con la sua ossessione per le comunità violate, il sangue dei martiri, l'onore degli eroi e delle eroine, l'empietà dei traditori, la crudeltà dei nemici. Il saggio di Pinto può condurre a trattare in classe il fenomeno del neoborbonismo non solo come vacua mistificazione nostalgica, ma anche strumento di insidiosa manovra politica, volta a sfruttare l'odierno disagio della società meridionale.

Avventurieri o martiri, malviventi o difensori dei più deboli, i briganti e la loro iconografia esercitano ancora un fascino misterioso. Molto spesso si è trattata di un'elaborazione mitizzante che non trova fattuali riscontri storici.

Obiettivo della scuola è lavorare sull'educazione civica ragionando di legalità e sfatando il falso mito del fuorilegge-eroe. Il libro di Pinto, togliendo ogni aura di eroismo ai briganti, è assai utile a questo scopo.

Altre fonti per approfondire

- F. Benigno, La mala setta: alle origini di mafia e camorra, 1859-1878, Torino, Einaudi, 2015

- S. Lupo, La mafia: Centosessant'anni di storia. Tra Sicilia e America, Roma, Donzelli, 2018

- E. Cinnella, Carmine Crocco. Un brigante nella grande storia, Pisa-Cagliari, Della Porta, 2010.

- A. De Jaco, Il brigantaggio meridionale. Storia inedita dell'unità di Italia, Roma, Editori Riuniti, 2005

- E. Felice, Perché il Sud è rimasto indietro, Bologna, il Mulino, 2013.

- L. Fiore, Briganti, Torino, UTET, 2017

- $\quad$ L. Fiore, L'ultimo re di Napoli, Torino UTET, 2018

- G.L. Fruci, C. Pinto, Neoborbonismo e sudismo, in "Il Mulino on line", 30 agosto 2017

- A. Mastropaolo, Le sirene del neoborbonismo, in "il Mulino on line", 8 settembre 2017

Note:

[1] C. Pinto, La guerra per il Mezzogiorno. Italiani, borbonici e briganti 1860-1870, Laterza, Bari 2019, prefazione

[2]Pinto, 2019, pag. 3

[3] Termine usato un G.L. Fruci, C. Pinto, Neoborbonismo e sudismo, in "Il Mulino on line", 30 agosto 2017 